ISSN 0103-5150

Fisioter. Mov., Curitiba, v. 26, n. 1, p. 123-131, jan./mar. 2013

Licenciado sob uma Licença Creative Commons

\title{
A eletroestimulação pode ser considerada uma ferramenta válida para desenvolver hipertrofia muscular?
}

\author{
Can electrical stimulation be considered a valid tool to \\ develop muscle hypertrophy?
}

\author{
Andrei Pereira Pernambuco ${ }^{[a]}$, Natane Moreira de Carvalho ${ }^{[b]}$, Aladir Horácio dos Santos ${ }^{[c]}$ \\ [a] Fisioterapeuta, doutorando em Biologia Celular na Universidade Federal de Minas Gerais (UFMG), professor do Centro \\ Universitário de Formiga (Unifor), Formiga, MG - Brasil, e-mail: pernambucoap@ymail.com \\ [b] Enfermeira, especialista em Terapia Intensiva pela Universidade Federal de Minas Gerais (UFMG), preceptora de estágios \\ da Universidade de Itaúna (UIT), Itaúna, MG - Brasil, e-mail: natanecarvalho@gmail.com \\ [c] Doutor em Agronomia (Estatística e Experimentação Agropecuária) pela Universidade Federal de Lavras (UFLA), docente \\ do Centro Universitário de Formiga (Unifor), Formiga, MG - Brasil, e-mail: aladirhs@oi.com.br
}

\section{Resumo}

Introdução: Aparelhos de estimulação elétrica, dentre eles a Corrente Russa (CR), vêm sendo cada vez mais utilizados por leigos e profissionais da saúde que objetivam aumentar a força e o volume muscular, porém são escassos e contraditórios os estudos relacionados a esse tipo de recurso na literatura atual. Objetivo: Avaliar se a estimulação elétrica com CR é capaz de gerar hipertrofia muscular no músculo reto abdominal. Materiais e métodos: Vinte e uma voluntárias saudáveis foram submetidas a 30 sessões de estimulação elétrica com CR sobre o ventre superior do músculo reto abdominal (VSMRA). As participantes também foram submetidas a exames de ultrassonografia antes e imediatamente após a intervenção, com a finalidade de mensurar a espessura desse músculo. Para o tratamento estatístico dos dados, utilizou-se a estatística descritiva com mediana, média e desvio padrão, além do teste $t$ pareado com um nível de significância de $\alpha=0,05$. Resultados: Foram observados pequenos aumentos da espessura do músculo avaliado em algumas das voluntárias, entretanto, eles não resultaram em significância estatística. Conclusão: Ao término do estudo, verificou-se que a CR não associada a exercícios físicos regulares é incapaz de promover hipertrofia muscular após 30 sessões.

Palavras-chave: Hipertrofia. Músculos. Estimulação elétrica. Ultrassonografia. 


\section{Abstract}

Introduction: Electrical stimulation devices, including the Russian Current $(R C)$, have been increasingly used by lay people and health professionals that aim to increase strength and muscle volume, however, the studies related to this type of resource are scarce and contradictory in the current literature. Objective: To assess whether electrical stimulation $R C$ is capable of generating muscular hypertrophy in the rectus abdominal. Materials and methods: Twenty-one healthy volunteers were submitted to 30 sessions of electrical stimulation with $R C$ on of the superior womb of the rectus abdominal muscle (SWMRA). Participants also underwent ultrasonography examinations before and immediately after the intervention with the purpose of measuring muscle thickness. For the statistical analyses, we used descriptive statistics with average, median and standard deviation, besides the paired T-test with a significance level of $\alpha=0.05$. Results: Small increases were observed in muscle thickness measured in some of the volunteers; however, these did not result in statistical significance. Conclusion: The study showed that the RC not associated with regular physical exercise is unable to promote muscle hypertrophy.

Keywords: Hypertrof. Muscles. Electrical stimulation. Ultrasonography.

\section{Introdução}

A busca pela forma física ideal e as novas tecnologias propiciadas pela bioengenharia e eletrofisiologia vêm provocando um aumento indiscriminado na utilização de produtos denominados eletroestimuladores musculares. Esses produtos prometem diversificados benefícios, como diminuição da gordura corporal, tonificação muscular, redução de medidas, fortalecimento e hipertrofia muscular; como contrapartida, exigem do usuário apenas alguns minutos de seu tempo $(1,2,3,4)$. As facilidades prometidas atraem a atenção das pessoas, já que a sobrecarga de tarefas no dia a dia vem se tornando um empecilho à realização de atividades físicas, pois elas, na maioria das vezes, consomem muito tempo de seus praticantes (5).

Em razão desses possíveis benefícios, os aparelhos de eletroestimulação conquistam mais consumidores a cada dia. Tal fato também pode ser verificado entre os profissionais da reabilitação, que estão cada vez mais incorporando esses recursos à sua prática clínica, apesar de muitas vezes não se basearem em evidências científicas $(2,4,6)$.

Embora sejam amplos os efeitos fisiológicos prometidos, ainda são escassos e contraditórios os estudos na literatura sobre os reais benefícios das correntes elétricas sobre o músculo estriado esquelético. Tais divergências ocorrem principalmente pela não padronização da metodologia dos estudos, pelos diferentes aparelhos eletroestimuladores utilizados ou, ainda, pela incoerência na seleção dos parâmetros da corrente $(2,4,6,7,8,9)$.

Cinco tipos de correntes elétricas podem ser encontrados no mercado: Estimulação Muscular Eletrônica (EMS); Estimulação Muscular Russa; Estimulação Elétrica Nervosa Transcutânea (TENS); Estimulação Interferencial e Eletroacupuntura (10). É válido ressaltar que, para alguns autores, a Corrente Russa (CR) faz parte da EMS (2, 7).

Dentre os efeitos prometidos, o que mais atrai a atenção dos fisioterapeutas, educadores físicos e leigos é a promessa de se obter a hipertrofia muscular sem necessitar de treinamentos intensos com cargas elevadas e grande número de repetições (4, $5,10)$. 0 processo de hipertrofia muscular é definido como o aumento na secção transversa do músculo, resultante do aumento do tamanho e do número de filamentos de actina e miosina com consequente adição de sarcômeros em paralelo dentro das fibras musculares já existentes e que resulta em aumento da força contrátil (11). Sendo assim, em dois músculos iguais, o músculo mais forte é o que possui maior diâmetro de secção transversa (12).

Segundo algumas evidências, a CR é capaz de combater a gordura localizada e gerar fortalecimento muscular, e por isso está amplamente difundida entre as clínicas de fisioterapia, de estética e entre usuários de academias de ginástica $(13,14)$. Essa corrente elétrica, além de ser relativamente agradável, dificilmente fere a pele; ainda assim, quando usada com intensidade adequada, causa grande 
tensão no músculo, recrutando maior número de unidades motoras (7), motivo pelo qual foi selecionada para este estudo.

A CR é considerada uma corrente elétrica de média frequência $(2.000$ a $10.000 \mathrm{~Hz}$ ), entretanto, como as frequências entre 2.000 e $2.500 \mathrm{~Hz}$ produzem maior atividade motora e são mais agradáveis aos usuários, essa faixa de frequência vem sendo utilizada de forma padrão nos atuais modelos de aparelhos de CR (7, 15). Esse tipo de corrente é formado por "trens de pulso" do tipo retangulares ou senoidais, bipolares, simétricos e emitidos numa frequência de $2.500 \mathrm{~Hz}$, modulada por uma onda que pode variar de $50 \mathrm{a} 80 \mathrm{~Hz}$ e é utilizada com fins excitomotores $(7,15,16)$.

Dentro do contexto abordado, o objetivo deste estudo foi verificar se a CR não associada a exercícios resistidos é capaz de gerar hipertrofia no ventre superior do músculo reto abdominal (VSMRA).

\section{Materiais e métodos}

Este estudo de caráter longitudinal e intervencional foi desenvolvido nos meses de dezembro de 2009 a junho de 2010 e obedeceu a todas as recomendações da Resolução n. 196/96 do CNS/MS (17). 0 protocolo de pesquisa foi aprovado pelo comitê de Ética em Pesquisa em Humanos do Centro Universitário de Formiga (COEPH/Unifor-MG) através do Parecer n. 101/2009.

Para a participação na pesquisa, os voluntários deveriam atender aos seguintes critérios de inclusão: ser do gênero feminino, ter idade entre 18 e 30 anos e assinar o Termo de Consentimento Livre e Esclarecido (TCLE). Os seguintes critérios de exclusão foram adotados para a execução da pesquisa: presença de obesidade (IMC > 30); hematoma e/ou escoriações na região abdominal; dores pélvicas e ou abdominais; procedimento de laparotomia há menos de dois anos; gravidez ou lactação; deficiência ou ausência de sensibilidade cutânea; processo infeccioso e ou inflamatório na região abdominal; e ausência em qualquer uma das sessões de eletroestimulação.

Participaram da pesquisa 21 indivíduos do sexo feminino, com idade entre 19 e 27 anos $(22,66 \pm 2,39)$, altura entre 1,58 e $1,72 \mathrm{~m}(1,66 \pm 0,04)$, peso entre 50 e $70 \mathrm{~kg}(60,09 \pm 5,08)$ e IMC entre 19,83 e 24,91 $(21,74 \pm 1,53)$.

Antes da realização da intervenção, todas as participantes foram submetidas a um exame de ultrassonografia diagnóstica, com a finalidade de determinar a espessura do VSMRA. Em seguida, os sujeitos da pesquisa foram submetidos a 30 sessões de estimulação com CR, sobre o músculo reto abdominal (direito e esquerdo); cada sessão teve duração de 15 minutos e foram realizadas cinco vezes por semana, durante seis semanas consecutivas (18).

Para as sessões de eletroestimulação, utilizou-se um equipamento Kinesis Corrente Russa (KW Indústria Nacional de Tecnologia LTDA, Amparo, SP, Brasil), com certificado de calibração válido no período da pesquisa. Os parâmetros da corrente utilizada no protocolo de atendimento foram: frequência $($ rate $)=50$ Hz; tempo de subida (rise) = três segundos; tempo de permanência (cicle on) = nove segundos; tempo de descida (decay) = três segundos; tempo de repouso (cicle off $)=$ nove segundos. A intensidade utilizada foi a máxima suportada por cada uma das participantes do estudo (18). Durante o procedimento de estimulação, todas as participantes permaneceram em decúbito dorsal, com flexão dos quadris e joelhos, e com os pés apoiados sobre a maca.

A estimulação foi proporcionada por quatro eletrodos (dois canais), sendo um eletrodo na porção superior do reto abdominal, sobre o ponto motor identificado manualmente e outro na porção média do mesmo músculo, procedimento realizado bilateralmente.

Ao término das 30 sessões de eletroestimulação, as participantes foram submetidas a outro exame de ultrassonografia do VSMRA.

Para o tratamento estatístico dos dados, utilizou-se estatística descritiva com média, mediana e desvio padrão. Também foi aplicado o teste $t$ pareado com um nível de significância ajustado para $\alpha=0,05$.

\section{Resultados}

Após a realização do teste $t$ pareado para verificar a diferença entre as médias das medidas obtidas no ventre muscular dos lados, direito e esquerdo, não houve diferenças significativas entre as médias iniciais e finais. Portanto, com base nos resultados obtidos neste estudo, rejeita-se a hipótese de que a CR não associada a exercícios resistidos possa gerar hipertrofia muscular, já que o valor de p encontrado foi superior a $0,05(>0,423)$, conforme se verifica na Tabela 1. 
Tabela 1 - Média, mediana, desvio padrão e valor de p para as diferenças pré e pós-intervenção para lado direito e esquerdo

\begin{tabular}{lccccc}
\hline & Voluntárias $(\mathbf{n})$ & Média & Mediana & Desvio padrão & $\mathbf{p}$ \\
\hline Direito antes & 21 & 1,07 & 1,06 & 0,03 & \\
Direito depois & 21 & 1,08 & 1,08 & 0,03 & \\
Diferença & & 0,01 & 0,02 & 0,00 & $0,054^{*}$ \\
& & & & & \\
Esquerdo antes & 21 & 1,06 & 1,06 & 0,03 & \\
Esquerdo depois & 21 & 1,07 & 1,07 & 0,03 & \\
Diferença & & 0,01 & 0,01 & 0,00 & $0,056^{*}$ \\
\hline
\end{tabular}

Legenda: ${ }^{*}=p>0,05$.

Fonte: Dados da pesquisa.

Ao se observar a espessura média do VSMRA, antes e após a intervenção, do lado esquerdo e do lado direito, é possível constatar acréscimos nos valores. Entretanto, eles não resultaram em significância estatística (Gráfico 1).

Com relação às medidas individuais, verificou-se que o VSMRA do lado direito permaneceu com medidas inalteradas durante a mensuração pré e pós-intervenção em 17 das 21 participantes, e que em quatro delas ocorreu um aumento médio de $0,07 \mathrm{~cm}$ (Gráfico 2).

No que se refere à espessura do VSMRA do lado esquerdo, situação semelhante pôde ser observada.

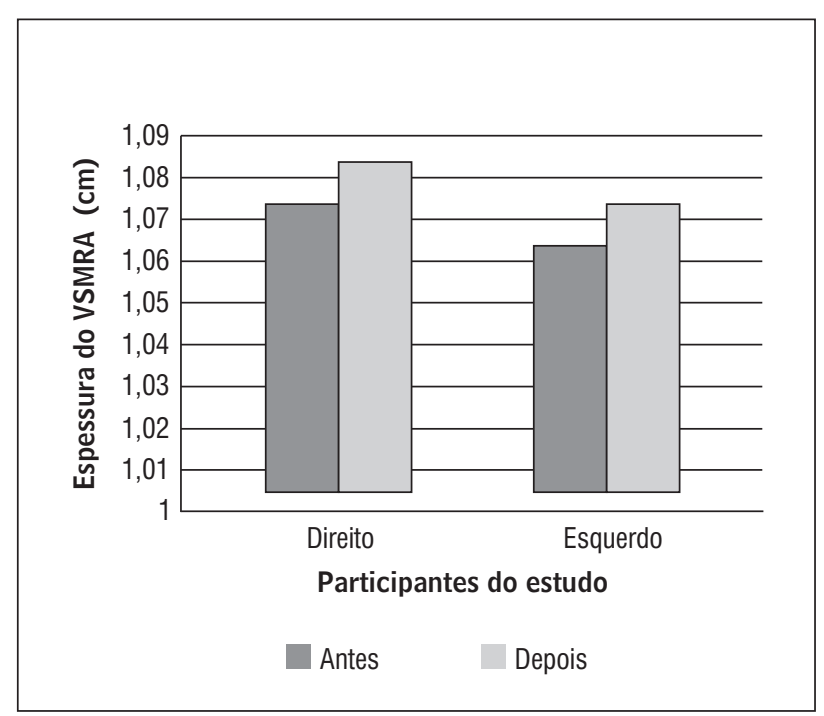

Gráfico 1 - Média da espessura do VSMRA, observada nos lados direito e esquerdo, antes e após trinta sessões de estimulação com Corrente Russa

Fonte: Dados da pesquisa.
Medidas inalteradas, antes e depois da estimulação com CR, foram encontradas nas mesmas 17 participantes, e entre quatro voluntárias novamente ocorreram acréscimos com média de 0,07 cm (Gráfico 3).

\section{Discussão}

0 músculo reto abdominal se localiza na parede anterior do abdome, separado de seu homônimo pela linha Alba. Classificado morfologicamente como um músculo poligástrico, possui quatro ventres musculares separados por intersecções tendíneas. Sua origem no corpo do osso púbis e sua inserção na quinta, sexta e sétima cartilagens costais, e também no processo xifoide, permitem que o reto abdominal realize a flexão anterior de tronco, auxilie na sustentação da coluna e na expiração forçada, além de auxiliar na contenção do conteúdo abdominal (12).

Neste estudo, cerca de um quinto das participantes apresentaram acréscimo na espessura do VSMRA direito e esquerdo após estimulação com CR. Tal fato pode estar relacionado à prática de exercícios físicos logo após o término das sessões de eletroestimulação. Essa hipótese é levantada, pois ao se analisar as características da amostra e suas variáveis, nota-se que apenas as voluntárias que praticavam atividade física com frequência de, pelo menos, três vezes por semana obtiveram acréscimos na espessura do VSMRA. A atividade física associada à eletroestimulação de fato incrementa o ganho de força e proporciona hipertrofia muscular em menor tempo do que o exercício físico tradicional $(10,13,14,15,19)$. 


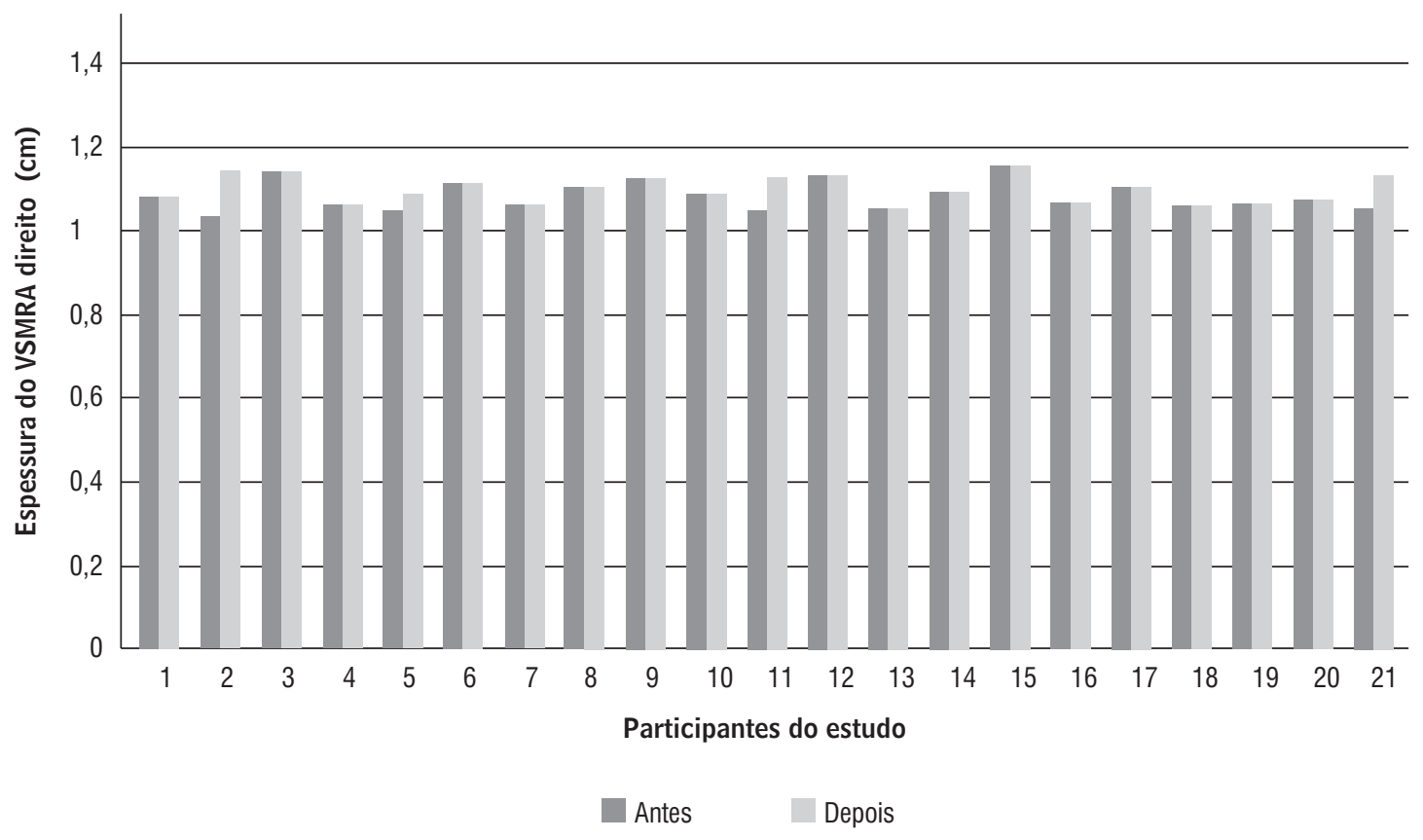

Gráfico 2 - Espessura do VSMRA direito de cada uma das participantes, antes e após 30 sessões de estimulação com Corrente Russa Fonte: Dados da pesquisa.

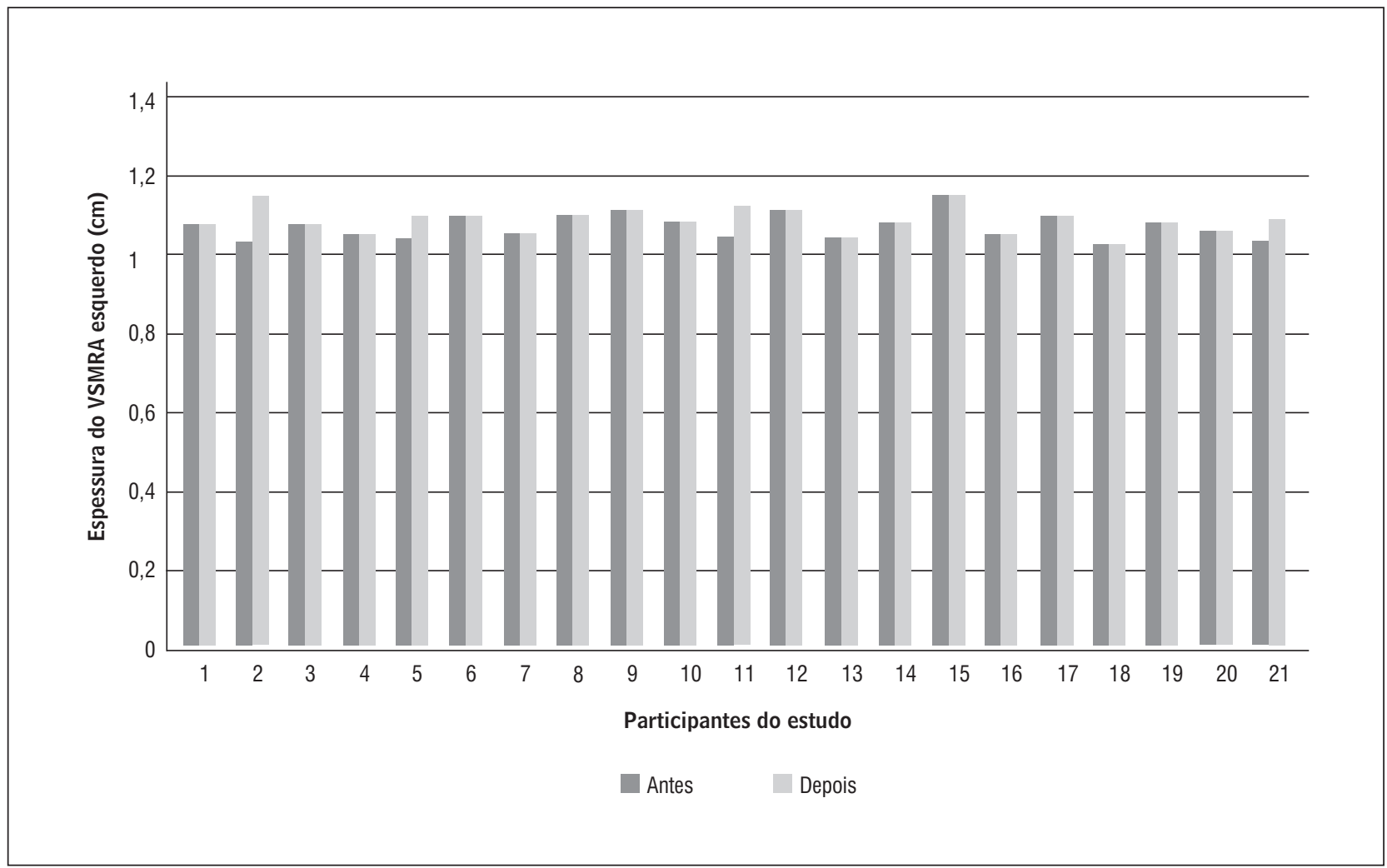

Gráfico 3 - Espessura do VSMRA esquerdo de cada uma das participantes, antes e após 30 sessões de estimulação com Corrente Russa

Fonte: Dados da pesquisa. 
Tais ganhos podem ser de $30 \%$ a $40 \%$ maiores em atletas (15).

Os resultados de Silva et al. (19) atestaram a eficácia da eletroestimulação russa enquanto método coadjuvante para ganho de força muscular. Os autores evidenciaram, após treinamento de oito semanas, que indivíduos que realizaram exercícios ativos resistidos, associados à CR, obtiveram ganho significativo na força muscular do quadríceps, quando comparado ao grupo controle, que somente realizou exercícios físicos.

Em relação às participantes que mantiveram suas medidas, as evidências deste estudo sugerem que a CR não associada a exercícios físicos é incapaz de promover hipertrofia muscular. Esses achados corroboram com os de Matias e Castro (10), que afirmam que a eletroestimulação possui resultados comprovados no controle de dor, reabilitação e reeducação muscular, redução de espasmos e, sobretudo, na prevenção de atrofias. Porém, em relação ao ganho de força e hipertrofia, estas somente seriam possíveis em associação com exercícios resistidos.

Os resultados de outros estudos também corroboram nossos achados, visto que não evidenciaram aumento da força e do volume muscular após utilização de diferentes tipos de correntes elétricas em diferentes grupos de pacientes $(2,3,4,5,6,20,21,22)$. Ávila et al. (2), ao pesquisarem os efeitos da CR sobre 20 jovens sadios, inferiram que a corrente elétrica, mesmo associada a um treinamento isocinético, é incapaz de gerar aumentos significativos tanto na força muscular, como em outras propriedades neuromusculares. Também com o objetivo de verificar os efeitos da estimulação elétrica sobre a força muscular, Bily et al. (3) realizaram estimulação elétrica durante 20 minutos, duas vezes ao dia, cinco vezes por semana, durante 12 semanas e também não observaram melhora da força muscular em quadríceps de pacientes portadores de síndrome da dor patelofemoral. Contudo, esses autores verificaram resultados significativos no que se refere à redução da dor.

Da mesma maneira, de Kroon e IJzerman (20), ao realizarem um ensaio clínico randomizado para verificar a eficácia da EMS sobre 22 pacientes acometidos por acidente vascular encefálico (AVE) crônico, também não observaram diferenças significativas entre o grupo intervenção e o grupo controle em relação à força muscular. Grillo e Simões (5), por sua vez, utilizaram dois protocolos para ganho de força e verificaram que o grupo submetido a exercícios resistidos obteve melhora significativa de força quando comparado com o grupo submetido à eletroestimulação. Esses achados corroboram os de Santos et al. (4), que analisaram a eficácia de exercícios físicos vs. a utilização de aparelhos domésticos de eletroestimulação para ganhos de força muscular, concluindo que só foram detectados aumentos significativos na força muscular e no trofismo muscular no grupo que realizou exercícios ativos. Dessa maneira, questionam a eficácia dos aparelhos eletroestimuladores de uso doméstico.

McLoughlin et al. (21) testaram os efeitos de uma corrente elétrica monofásica sobre lesões musculares, e não observaram diferenças significativas na força nem no trofismo muscular dos voluntários. Pomeroy et al. (6), ao realizarem uma revisão sistemática da literatura referente ao uso da EMS sobre o movimento e habilidades funcionais de pacientes com AVE, concluíram que existe uma grande heterogeneidade metodológica entre os estudos e que somente os que utilizaram placebo no grupo controle obtiveram resultados positivos.

Por sua vez, Tong et al. (22), ao utilizarem o FES (Estimulação Elétrica Funcional) cinco minutos por dia, cinco dias por semana, durante quatro semanas, para fins de melhora da marcha em pacientes na fase subaguda do AVE, também não encontraram evidências de que o FES contribuísse para a melhora da velocidade da marcha, funcionalidade e mobilidade do paciente.

Porém, diversas pesquisas contestam nossos achados, no momento em que constataram aumento na força muscular e ou no desempenho de indivíduos submetidos à eletroestimulação $(8,18,23-32)$.

Utilizando-se de protocolo semelhante ao deste estudo, Briel et al. (18) estimularam os músculos flexores de punho no antebraço não dominante de 15 indivíduos saudáveis. Ao final de 30 dias, os autores constataram aumentos significativos no ganho de força e no trofismo muscular.

Powell et al. (27) e Kimberley et al. (28), ao realizarem ensaios clínicos randomizados para investigarem o aumento da força muscular isométrica durante a extensão do punho em pacientes que haviam sofrido AVE, encontraram fortes indícios de que a eletroestimulação consegue elevar os níveis de força em isometria. Do mesmo modo, Yozbatiran et al. (32) verificaram melhoras significativas na função da mão de pacientes hemiplégicos após serem tratados com a técnica de Bobath associada à SEM. Tais ganhos foram significativamente maiores que os obtidos pelo grupo tratado somente 
com a técnica de Bobath, entretanto, foram possíveis ao associar a EMS a uma técnica de movimentação ativa.

Avramidis et al. (23) e Buhmann et al. (8) fizeram uso da EMS no pós-cirúrgico de artroplastia de quadril e de reconstrução do ligamento cruzado anterior, respectivamente. Os primeiros autores encontraram melhora na velocidade da marcha após estimularem o músculo vasto medial; já Buhmann et al. (8) constataram que a EMS melhorou a força muscular medida pelo dinamômetro isocinético e a atividade muscular medida por meio de miofeedback.

Callaghan et al. (24) investigaram os efeitos de dois diferentes tipos de correntes elétricas sobre a recuperação do trofismo do quadríceps em pacientes com síndrome da dor patelofemoral e encontraram indícios de que ambas as correntes testadas foram capazes de promover melhoras significativas nos dois grupos eletroestimulados. Os ganhos ocorreram no torque de extensão isométrica e isotônica, bem como na redução da fadiga e da dor. Além disso, os autores ainda verificaram melhoras no que se refere à função e ao diâmetro de secção transversa do quadríceps, aferidas por perimetria.

Os achados de Nunes et al. (13) também apontam para benefícios proporcionados pela SEM. Os autores avaliaram a resposta de dez crianças espásticas submetidas a 30 minutos de estimulação elétrica duas vezes por semana, sobre o músculo tibial anterior, e verificaram aumento da força muscular, da amplitude de movimento e da função motora grossa. Gerovasili et al. (30), ao se utilizarem de metodologia semelhante à utilizada em nosso estudo, tentaram observar o efeito da EMS sobre a redução da atrofia em pacientes criticamente enfermos. Ao final do estudo, os autores verificaram, pelo uso de ultrassonografia diagnóstica, redução significativa da atrofia muscular no grupo eletroestimulado, demonstrando que a eletroestimulação pode ser eficiente para prevenir atrofias.

Os resultados de Ward e Shkuratova (31) também discordam de nossos achados, já que os autores verificaram ganhos de até $45 \%$ na força muscular e de $3 \%$ na circunferência de membros eletroestimulados. Robinson e Mackler (28) também verificaram ganhos de $20 \%$ na força muscular após um mês de eletroestimulação; segundo os autores, são atribuídos ao recrutamento de $30 \%$ a $40 \%$ a mais de unidades motoras, ocasionadas pela eletroestimulação de média frequência.

Soares et al. (29), por sua vez, constataram que a corrente de média frequência é mais eficaz no aumento de força muscular do que as correntes de baixa frequência, apesar de ambas terem produzido ganho de força. Segundo os autores, as correntes de média frequência podem gerar um aumento de força de $22,7 \%$, e as de baixa frequência, aumento de até 8,7\%. Em estudo semelhante, Nunes (26) encontraram aumento significativo da força muscular nos grupos eletroestimulados com correntes de média e baixa frequência. Lucena (33) afirma que grupos musculares eletroestimulados são capazes de incrementar seu percentual de força, acima do percentual alcançado pelos grupos musculares submetidos a exercícios resistidos.

Enfim, nota-se que pesquisas realizadas com o intuito de verificar a ação das correntes elétricas sobre a força, torque e tônus muscular, vêm apresentando resultados díspares. Tal fato está possivelmente relacionado à diversidade de protocolos metodológicos propostos, à grande variedade de correntes elétricas utilizadas, a diferenças na seleção dos parâmetros da corrente, ao número de sessões realizadas, ao grupo muscular estimulado, dentre outros, o que resulta em controvérsias nas conclusões enunciadas pelos experimentos.

\section{Conclusão}

Os achados deste estudo sugerem que CR não associada à prática de atividade física regular é incapaz de promover hipertrofia muscular no músculo reto abdominal.

Acredita-se que nossos achados possam contribuir para o pensamento e tomada de decisão clínica de profissionais que utilizam a CR como forma de tratamento, além de informar aos potenciais consumidores desse tipo de dispositivo os seus reais benefícios.

Mais estudos com objetivos semelhantes se fazem necessários, uma vez que futuras investigações poderão esclarecer inconsistências observadas na literatura.

\section{Referências}

1. Kantor G, Alon G, Ho HS. The effects of selected stimulus waveforms on pulse and phase characteristics at sensory and motor thresholds. Phys Ther. 1994;74(10):951-62. PMid:8090846. 
2. Avila MA, Brasileiro JS, Salvini TF. Electrical stimulation and isokinetic training: effects on strength and neuromuscular properties of healthy young adults. Rev Bras Fisioter. 2008;12(6):435-40. doi:10.1590/ S1413-35552008005000006.

3. Bily W, Trimmel L, Modlin M, Kaider A, Kern H. Training program and additional electric muscle stimulation for patellofemoral pain syndrome: a pilot study. Arch Phys Med Rehabil. 2008;89(7):1230-6. doi:10.1016/j.apmr.2007.10.048.

4. Santos FMI, Rodrigues RGS, Trindade-Filho EM. Exercício físico versus programa de exercício pela eletroestimulação com aparelhos de uso doméstico. Rev Saúde Pública. 2008;42(1):117-22. doi:10.1590/ S0034-89102008000100015.

5. Grillo DE, Simões AC. Atividade física convencional (musculação) e aparelho eletroestimulador: um estudo da contração muscular. Estimulação elétrica: mito ou verdade? Rev Mackenzie Educ Fís Esporte. 2003;2(2):31-43.

6. Pomeroy VM, King L, Pollock A, Baily-Hallam A, Langhorne P. Electrostimulation for promoting recovery of movement or functional ability after stroke. Cochrane Database Syst Rev. 2006;37:2441-2.

7. Agne JE. Eletrotermoterapia teoria e prática. Santa Maria: Orium; 2005.

8. Buhmann HW, Schleicher W, Urbach D, Schultz W. Electromyostimulation and isokinetic training in rehabilitation after anterior cruciate surgery. Physikalische Medizin, Rehabilitationsmedizin, Kurortmedizin. 1998;8(1):13-6. doi:10.1055/s-2008-1061813.

9. Kitchen S, Bazin S. Eletroterapia de Clayton. São Paulo: Manole; 1998.

10. Matias R, Castro N. Estimulação electromuscular: o futuro do exercício? Estimulação Electromuscular. Proc. Workshop BioMed, 2002 [acesso 30 jan 2013]. Disponível em: http://atletismo.files.wordpress. com/2007/04/estimelectromuscular.pdf.

11. Bompa TO. Treinamento de força consciente. São Paulo: Phorte; 2000.

12. Dangelo, JG, Fattini CA. Anatomia humana sistêmica e segmentar: para o estudante de medicina. São Paulo: Editora Atheneu; 2002.
13. Nunes LCBG, Quevedo A, Magdalon E. Effects of neuromuscular electrical stimulation on tibialis anterior muscle of spastic hemiparetic children. Rev Bras Fisioter. 2008;12(4):317-23. doi:10.1590/ S1413-35552008000400011.

14. Sivini SCL, Lucena ACT. Desenvolvimento da Força Muscular através da Corrente Russa em Indivíduos Saudáveis [dissertação]. Recife: Universidade Federal de Pernambuco; 1999.

15. Kots Y. Electrostimulation. Paper presented at Symposium on Electroestimulation on Skelet muscles, Canadian Soviet Exchange Symposium, Concordia University. 1977:6-10.

16. Low J, Red A. A eletroterapia aplicada - princípios e prática. São Paulo: Manole; 2001.

17. Ministério da Saúde. Conselho Nacional de Saúde. Resolução n. 196, de 1996. Normas para pesquisa envolvendo seres humanos [citado 30 jan. 2013]. Disponível em: http://bvsms.saude.gov.br/bvs/publicacoes/normas_pesquisa_sereshumanos.pdf.

18. Briel AF, Pinheiro MF, Lopes LG. Influência da corrente russa no ganho de força e trofismo muscular dos flexores no antebraço não dominante. Arq Ciênc Saúde Unipar. 2003;7(3):205-10.

19. Silva RT, Knorr LF, Lopes RF, Knorr L, Navarro F. Comparação entre os efeitos do uso de eletroestimulação neuromuscular associada ao treinamento de força com somente treinamento de força em exercício de membros inferiores durante oito semanas. RBPFEX. 2007;1(5):1-10.

20. de Kroon JR, Ijzerman MJ. Electrical stimulation of the upper extremity in stroke: cyclic versus EMG triggered stimulation. Clin Rehabil. 2008;22(8):690-7. doi:10.1177/0269215508088984.

21. McLoughlin TJ, Snyder AR, Brolinson PG, Pizza FX. Sensory level electrical muscle stimulation: effect on markers of muscle injury. Br J Sports Med. 2004; 38(6):725-9. doi:10.1136/bjsm.2003.007401.

22. Tong RK, Ng MF, Li LS. Effectiveness of gait training using an electromechanical gait trainer, with and without functional electric stimulation, in subacute stroke: a randomized controlled trial. Arch Phys Med Rehabil. 2006;87(10):1298-304.doi:10.1016/j. apmr.2006.06.016 
23. Avramidis K, Strike PW, Taylor PN, Swain ID. Effectiveness of electric stimulation of the vastus medialis muscle in the rehabilitation of patients after total knee arthroplasty. Arch Phys Med Rehabil. 2003;84(12):1850-3. doi:10.1016/S0003-9993(03)00429-5

24. Callaghan MJ, Oldham JA, Winstanley J. A comparison of two types of electrical stimulation of the quadriceps in the treatment of patellofemoral pain syndrome. A pilot study. Clin Rehabil. 2001;15(6):637-46.

25. Kimberley TJ, Auerbach EJ, Lojovich GM, Lewis SM, Dorsey LL, Carey JR. Electrical stimulation driving functional improvements and cortical changes in subjects with stroke. Exp Brain Res. 2004;154: 450-60.

26. Nunes CV. Efeito da estimulação elétrica neuromuscular na atividade eletromiográfica e na força dos músculos extensores da perna. Disponível em: http:// www.profala.com/artifisio8.htm.

27. Powell J, Pandyan AD, Granat M, Cameron M, Stott D. Electrical stimulation of wrist extensors in poststroke hemiplegia. Stroke. 1999;30(7):1384-89.

28. Robinson AJ, Mackler SL. Eletrofisiologia clinica: eletroterapia e teste eletrofisiológico. 2. ed. Porto Alegre: Artmed; 2001.

29. Soares AV, Pagliosa F, Oliveira GO. Estudo comparativo entre a estimulação elétrica neuromuscular de baixa e média freqüência para o incremento da força de preensão em indivíduos sadios não-treinados. Fisioter Bras. 2002;3(6):345-50.
30. Gerovasili V, Stefanidis K, Vitzilaios K, Karatzanos E, Papadopoulos E, Tzanis G, et al. Efficacy of electrical muscle stimulation on preserving the muscle critically ill patients. Crit Care. 2008;12(Suppl 2):516.

31. Ward AR, Shkuratova N. Russian electrical stimulation: the early experiments. Phys Ther. 2002;82(10):1019-30.

32. Yozbatiran N, Donmez B, Kayak N, Bozan O. Electrical stimulation of wrist and fingers for sensory and functional recovery in acute hemiplegia. Clin Rehabil. 2006;20(1):4-11.

33. Lucena C. Bases neurofisiológicas da eletroterapia. Curitiba: Levise; 1999.

Recebido: 10/01/2012

Received: 01/10/2012

Aprovado: 21/08/2012

Approved: 08/21/2012 